\title{
Desigualdades e fatores associados ao tratamento do problema crônico de coluna no Brasil
}

\author{
Chronic low back pain treatment in Brazil: inequalities \\ and associated factors
}

Dalia Elena Romero (https://orcid.org/0000-0002-2643-9797) ${ }^{1}$

Jéssica Muzy (https://orcid.org/0000-0003-2526-2317) ${ }^{1}$

Leo Maia (https://orcid.org/0000-0003-1531-0880) ${ }^{2}$

Aline Pinto Marques (https://orcid.org/0000-0002-9072-5333) ${ }^{1}$

Paulo Roberto Borges de Souza Júnior (https://orcid.org/0000-0002-8142-4790) ${ }^{1}$

Débora Castanheira (https://orcid.org/0000-0001-6995-1259) ${ }^{3}$

${ }^{1}$ Departamento de Informações para a Saúde, Fiocruz. Av. Brasil 4365, Manguinhos. 21040-900 Rio de Janeiro RJ Brasil. dalia.fiocruz@gmail.com ${ }^{2}$ Instituto de Comunicação e Informação Científica e Tecnológica em Saúde, Fiocruz. Rio de Janeiro RJ Brasil.

${ }^{3}$ Instituto de Estudos

Sociais e Políticos,

Universidade do Estado

do Rio de Janeiro. Rio de

Janeiro RJ Brasil.

\begin{abstract}
Chronic low back pain (LBP) is one of the most common diseases in the world and one of the leading causes of years of life lost due to disability. Despite being a major public health concern, studies on access to and use of different types of treatment are scarce. The aim of this article is to describe the most common treatments for chronic LBP in Brazil, examine the factors associated with the use of these treatments, and discuss possible inequalities in the use of physical therapy/exercise and medications. A descriptive analysis was performed using data from the 2013 National Health Survey. Multiple logistic regression was conducted to determine the association between treatment use and demographic, socioeconomic, health status, access to health services, and geographical characteristics. People with higher education were 2.39 times more likely to do physiotherapy. However, no association was found between education level and medication use. People in social class $A / B$ were almost twice as likely to do physical therapy. However, there was no association between social status and medication use. People with a very high or high degree of functional limitation were 3.5 times more likely to use medication. However, no association was observed between functional limitation and physical therapy use.
\end{abstract}

Key words Spine, Health inequalities, Physical therapy, Pharmacological treatment
Resumo O Problema Crônico de Coluna (PCC) é uma das doenças mais prevalentes no mundo e representa uma das principais causas de anos de vida perdidos por incapacidade. Embora de grande relevância para a saúde pública, estudos sobre o acesso e a utilização dos tipos de tratamento são escassos. O objetivo deste artigo é descrever os principais tratamentos para PCC no Brasil, analisar os fatores associados à sua utilização $e$ discutir possíveis desigualdades na utilização de fisioterapia /exercícios e a utilização de medicamentos. A partir da PNS 2013 foram realizadas análises descritivas sobre prevalência de PCC e regressão logística múltipla para analisar a associação segundo as características demográficas, socioeconômicas, a situação da saúde, a limitação causada pelo PCC, acesso a serviços de saúde e regionais. Ter ensino superior aumenta 2,39 vezes as chances de realizar fisioterapia, no entanto, a escolaridade não se mostrou associada à utilização de medicamentos. Indivíduos de estratos superiores apresentam quase 2 vezes mais chances de realizar fisioterapia, o que não foi observado para medicamentos. Sobre as condições de saúde, o aumento do grau da limitação por PCC eleva a chance de uso de medicamentos em até 3,5 vezes, mas não varia quando associado à realização de fisioterapia.

Palavras-chave Coluna vertebral, Desigualdades em saúde, Fisioterapia, Tratamento farmacológico 


\section{Introdução}

O Problema Crônico de Coluna (PCC) é considerado uma das doenças mais prevalentes no mundo e representa uma das principais causas de anos de vida perdidos por incapacidade, sendo a primeira em países de alta e média renda, como o Brasil ${ }^{1}$. A prevalência de PCC entre os adultos no Brasil, em 2013, foi de $18,5 \%{ }^{2-4}$, similar à observada em outros países ${ }^{5}$. O PCC é uma grande causa de busca por atendimento médico ${ }^{6,7}$, provoca graves perdas da qualidade da vida e é a principal causa de aposentadoria por invalidez ${ }^{8}$. Além disso, representa alto custo direto e indireto devido ao absenteísmo laboral, perda de produtividade $^{9}$ e pelas despesas com tratamentos como medicamentos, fisioterapia e cirurgias ${ }^{10}$.

Dados sobre a prevalência de PCC no Brasil estão disponíveis no Suplemento de Saúde da Pesquisa Nacional por Amostra de Domicílio (PNAD) desde 1998. Contudo, somente na Pesquisa Nacional de Saúde (PNS), de 2013, foram incluídas informação sobre idade de início do PCC, grau de limitação ocasionada pelo problema na coluna e tipos de tratamento realizados, possibilitando uma análise mais ampla desta enfermidade no Brasil. Apesar de relevantes publicações recentes abordarem o tema ${ }^{2-4}$, ainda não existem estudos representativos da população brasileira sobre a utilização de tratamentos para PCC.

A literatura internacional, aponta que as intervenções mais utilizadas são medicamentos e fisioterapia/exercícios. Enquanto os primeiros estão, em geral, relacionados a fases agudas do problema $^{11}$, os segundos são relacionados com práticas preventivas ${ }^{12}$. No Brasil, não existem estudos representativos que identifiquem os tipos de tratamento mais utilizados.

A importância de intervenções que previnam e controlem DCNT é bem solidificada na literatura, principalmente porque são elas que impedem o desenvolvimento de incapacidades funcionais nos indivíduos ${ }^{13}$. Podemos dizer, portanto, que a prática de fisioterapia/exercícios no controle do PCC é essencial para garantir a saúde da população. Revisões sistemáticas internacionais - uma com 6 estudos e outra com 35 - mostraram que a reabilitação envolvendo exercícios, educação e envolvimento ativo do paciente tem se mostrado eficaz na redução de limitações e incapacidades provocadas pelo PCC ${ }^{14,15}$. Apesar disso, estudos identificam que os medicamentos, principalmente analgésicos associados ao controle da dor, são a principal intervenção utilizada ${ }^{11,16}$.
Não existe consenso sobre as causas do maior uso de medicamentos. Porém, um estudo canadense de base populacional $(\mathrm{n}=113.229) \mathrm{de}-$ monstrou que a desigualdade socioeconômica explica em grande parte os diferenciais no acesso aos serviços e aos tipos de tratamento: pessoas de baixa escolaridade e status socioeconômico inferior utilizam menos os serviços de saúde para cuidados do PCC e o tipo de tratamento utilizado difere segundo sexo, idade e condições de saúde, como presença de depressão e outras comorbidades ${ }^{17}$.

No Brasil, os poucos estudos sobre tipos de tratamento do PCC disponíveis têm abrangência geográfica e populacional restrita. Um estudo realizado no município de Pelotas ( $\mathrm{n}=3100)$, mostrou que pessoas com melhor condição econômica utilizam mais fisioterapia, além de apontá-la como a principal recomendação médica para o tratamento dos problemas nas costas ${ }^{18}$. Outro estudo realizado no município de Belo Horizonte ( $n=76$ ), observou que $85,5 \%$ dos idosos com dor lombar não faziam uso do serviço de fisioterapia, um dos principais motivos apresentados foi a fila de espera ${ }^{19}$.

No Brasil, não existem estudos que analisem, a nível nacional, a partir de uma perspectiva epidemiológica, os tipos de tratamento para o PCC, assim como os fatores que explicam as possíveis desigualdades. Nesse sentido, este artigo tem como objetivo descrever os principais tratamentos para PCC no Brasil, analisar os fatores associados à sua utilização e discutir possíveis desigualdades na utilização de fisioterapia/ exercícios (como proxy de práticas preventivas) e a utilização de medicamentos (como proxy de intervenção em fase aguda).

\section{Metodologia}

\section{Fonte de informação}

Este estudo utilizou microdados da Pesquisa Nacional de Saúde (PNS), de 2013, um inquérito de base domiciliar conduzido pela Instituto Brasileiro de Geografia e Estatística (IBGE) cujo objetivo central é caracterizar a situação de saúde e estilo de vida da população, além de coletar informações sobre atenção à saúde, acesso e serviços de saúde ${ }^{20}$.

A Pesquisa tem delineamento amostral complexo por conglomerado em três estágios de seleção (setores, as famílias e indivíduos). No primeiro estágio, a seleção das unidades primárias 
de análise (UPA) foi obtida por amostragem aleatória simples (AAS) selecionadas previamente na Amostra Mestra. No segundo estágio, foi selecionado - por AAS - um número fixo de domicílios particulares permanentes (DPP) em cada UPA selecionada no primeiro estágio. No terceiro estágio, dentro de cada domicílio da amostra, um morador com 18 anos ou mais de idade foi selecionado - também por AAS - para responder à $3^{\mathrm{a}}$ parte (individual) do questionário.

O questionário foi dividido em três partes, as duas primeiras destinadas a perguntas sobre características do domicílio, situação socioeconômica e de saúde dos moradores; a terceira é individual e direcionada ao morador de 18 anos ou mais previamente selecionado, nela são respondidas perguntas sobre morbidade e estilo de vida $^{21}$. Essa seleção foi feita a partir de uma lista de moradores elegíveis, construída no momento da entrevista ${ }^{20}$. Para análise das prevalências de PCC foi utilizada a amostra total da PNS de 60202 pessoas maiores de 18 anos, já para a análise da utilização de tratamento do PCC a amostra abrange apenas as pessoas que autodeclararam ter problema crônico de coluna, um universo de 11118 indivíduos.

\section{Variáveis}

O PCC foi definida a partir da pergunta $O(A)$ sr(a) tem algum problema crônico de coluna, como dor crônica nas costas ou no pescoço, lombalgia, dor ciática, problemas nas vértebras ou disco?. As alternativas de respostas eram dicotômicas (Sim ou Não).

O tipo de tratamento realizado para o PCC obteve-se a partir da pergunta: $O$ que o(a) sr. (a) faz atualmente por causa do problema na coluna? As respostas incluem tanto as terapêuticas, orientadas por um profissional da saúde, como as de iniciativa do próprio paciente. Classificam-se em quatro alternativas, com a possibilidade de selecionar mais de uma resposta: 1 . Exercício ou fisioterapia 2. Usa medicamentos ou injeções. 3. Faz acupuntura 4. Outro (Especifique). No intuito de comparar as práticas de tratamento do PCC, neste trabalho, as respostas foram reagrupadas da seguinte forma: nenhum; só fisioterapia/exercícios, só medicamentos; fisioterapia/ exercícios e medicamentos e só acupuntura ou outros.

As variáveis demográficas utilizadas na análise foram: sexo (masculino, feminino) e idade (18 a 49 anos, 50 a 59 anos, 60 anos e mais). As variáveis socioeconômicas foram: escolarida- de (sem escolaridade, fundamental incompleto, fundamental completo, ensino médio completo, superior ou mais), cor/raça (branco, pardo, preto ou amarela ou indígena) e o estrato social que foi analisado a partir da definição do critério Brasil da Associação Brasileira de Empresas de Pesqui$\mathrm{sa}^{22}$ (D/E, B, A/B).

As variáveis relacionadas à situação de saúde analisadas foram: autoavaliação da saúde (muito ruim ou ruim, regular, boa, muito boa), intensidade das limitações por PCC (muito intensa ou intensa, moderada, pouco, não limita), depressão (ter depressão, não ter depressão) e número de comorbidade crônica (ter uma comorbidades, ter duas comorbidades, ter 3 ou mais comorbidades).

As variáveis de acesso a serviços de saúde foram: domicílio cadastrado na Estratégia Saúde da Família (ESF) (sim, não), possuir plano privado de saúde (sim, não). Grande região (Norte, Nordeste, Sudeste, Sul e Centro-Oeste) e local de residência (urbana, rural) foram utilizadas como variáveis regionais.

\section{Análise estatística}

A análise descritiva foi feita a partir da prevalência de PCC segundo características demográficas, socioeconômicas, situação da saúde, limitação causada pelo PCC, acesso a serviços de saúde e regionais. O tipo de tratamento para o PCC foi analisado a partir da distribuição percentual do tipo de intervenção segundo essas mesmas características.

Para o estudo da força de associação entre características demográficas, socioeconômicas, situação de saúde e tipos de tratamento foi utilizada a Razão de chances (OR) e intervalos de confiança de 95\% (IC95\%), calculados por meio de regressão logística. Para análise de associação foram definidas três variáveis dependentes: faz algum tratamento, faz fisioterapia/exercícios e uso de medicamento. Cada um dos três desfechos foi analisado a associação como as seguintes variáveis independentes: sexo, idade, escolaridade, raça/cor, estrato social, autoavaliação da saúde, depressão, comorbidade, intensidade da limitação por PCC, ESF, plano de saúde, área de residência e Grande Região de residência. Foram calculadas a razões de chance brutas (bivariadas) para os três desfechos e ajustadas (multivariadas) para os dois últimos desfechos por todas as variáveis independentes.

Por se tratar de uma pesquisa com desenho complexo de amostragem, com mais de um está- 
gio de seleção, todas as análises foram realizadas utilizando metodologia da biblioteca "complex sample" do software do Statistical Package for the Social Sciences (IBM SPSS 22).

\section{Resultados}

A prevalência de PCC no Brasil é de 18,5\% (IC95\% 17,8-19,1) $(\mathrm{n}=11118)$, variando por características demográficas, socioeconômicas, condições de saúde, acesso aos serviços e regionais. Mulheres têm maior prevalência de PCC que homens (21,1 IC95\% 20,2-21,9 e 15,5 IC 95\% 14,816,4 , respectivamente). A prevalência também aumenta gradativamente com a idade, chegando a $28,1 \%$ (IC95\% 26,6-29,7) entre a população idosa. Os brasileiros com condições socioeconômicas desfavoráveis e com piores condições da saúde têm maior prevalência de PCC (Tabela 1).

A Tabela 2 apresenta a distribuição dos portadores de PCC de acordo com os tipos de tratamento ou intervenção utilizados segundo algumas características da população. Quase metade das pessoas com PCC não realiza qualquer tipo de intervenção para tratamento do problema $(46,4 \%)$. Entre os homens, a proporção dos que não realizam intervenções é ligeiramente mais alta $(50,6 \%)$ em relação à das mulheres $(43,6 \%)$. Mulheres apresentaram cerca de $30 \%$ mais chance de realização de tratamento.

Pessoas com melhores condições socioeconômicas e os que têm piores condições de saúde apresentaram maior chance de realização de algum tratamento. Ter ensino superior aumenta as chances de realização de algum tratamento para PCC em 31\% (OR 1,31 IC95\% 1,05 - 1,64) e ser de estrato social A ou B aumenta em 24\% (OR 1,24 IC95\% 1,05 - 1,47). Pior autoavaliação de saúde, ter depressão, muitas comorbidades e limitações intensas causadas pelo PCC indicam a maior chance de realização de algum tipo de tratamento.

Em geral, o tipo de intervenção mais utilizado foi o uso de medicamento (40\%), com ou sem realização de outras intervenções combinadas. $\mathrm{O}$ uso de medicamento, sem a realização de fisioterapia/exercícios, foi de 31,6\% com pouca variação segundo sexo e idade. Quando analisada por características socioeconômicas, essa prática é maior entre pessoas sem escolaridade (40\%), de estrato social mais baixo $(38,5 \%)$ e pessoas não brancas $(33,5 \%)$. Segundo condições de saúde, a opção por esse tipo de tratamento é maior entre pessoas com autoavaliação de saúde ruim ou muito ruim $(43,4 \%)$, com depressão $(34,8 \%)$, com mais de três comorbidades $(38,9 \%)$, com muita limitação $(47,4 \%)$. Também foi encontrada maior proporção de pessoas utilizando apenas medicamentos entre os sem plano de saúde (35\%) e com domicílio cadastrado na ESF $(34,1 \%)$. Variáveis regionais mostraram que esse tipo de tratamento também é mais frequente em residentes da área rural (39\%) e nas regiões Norte $(34,2)$, Nordeste $(35,8$ e Centro-oeste $(34,5)$.

A prática de fisioterapia/exercício foi a segunda intervenção mais utilizada (18,8\%). Essa prática sem a utilização combinada de medicamento corresponde a $10,4 \%$ das pessoas que tratam o PCC. O uso de fisioterapia/exercícios como único tratamento é maior entre as pessoas de maior escolaridade - passando de 4,5\% entre os sem escolaridade até $23,1 \%$, entre pessoas com nível superior -, no estrato social A/B (17,8\%), da cor branca (12,6\%), com boa autoavaliação de saúde $(20,4 \%)$ e com plano de saúde $(17,9)$.

O uso concomitante de medicamento e fisioterapia/exercício como tratamento foi de $8,4 \%$, sendo mais alta para mulheres $(10,1 \%)$ do que para homens $(5,9 \%)$. A proporção de pessoas que realizam ambas as intervenções aumenta progressivamente com a melhora de condições socioeconômicas, tais como a escolaridade e estrato social, chegando a $10,6 \%$ para pessoas com ensino superior e $11,4 \%$ entre os de estrato social A ou B. A proporção também foi ligeiramente superior para pessoas da cor branca $(9,4 \%)$ quando comparados às não brancas $(7,5 \%)$.

$\mathrm{O}$ uso de medicamentos combinado à fisioterapia/exercícios também é maior entre os com piores condições de saúde, sendo 9,7\% entre os com autoavaliação de saúde ruim ou muito ruim, $12,7 \%$ entre pessoas com depressão, 12,5 entre os com 3 ou mais comorbidades e limitação intensa a muito intensa causada por PCC (12,9\%). Quanto ao acesso a serviços de saúde, medicamento e fisioterapia/exercícios foram utilizados por $9 \%$ dos que residem em domicílios cobertos pela ESF e 10,5\% entre os que possuem plano de saúde. Variáveis regionais evidenciaram diferenças quanto à realização de fisioterapia/exercícios, seja ela combinada ou não com uso de medicamentos, sendo mais baixa para as regiões Norte e Nordeste do que para as demais.

A utilização de acupuntura e outros tipos de intervenção sem utilização de medicamentos ou fisioterapia/exercícios é a menor entre os tratamentos analisados $(3,2 \%)$. Foram observadas poucas diferenças segundo características demográficas. Essas práticas são mais utilizadas 
Tabela 1. Características da população de 18 anos e mais, prevalência e distribuição do PCC segundo caracteristicas demográficas, socioeconômicas, condições de saúde, acesso a serviços de saúde, contextuais e grau de limitações. Brasil, 2013.

\begin{tabular}{|c|c|c|c|c|c|}
\hline & \multicolumn{2}{|c|}{ Amostra } & \multicolumn{3}{|c|}{ PCC } \\
\hline & $\mathbf{n}$ & $\%$ & $\mathbf{n}$ & $\begin{array}{c}\text { Prevalência } \\
\text { PCC }\end{array}$ & IC 95\% \\
\hline Total amostra & 60202 & 100,0 & 11118 & 18,5 & $17,8-19,1$ \\
\hline Total população brasileira & 146308458 & 100,0 & 27021122 & 18,5 & - \\
\hline \multicolumn{6}{|l|}{ Demográficos } \\
\hline \multicolumn{6}{|l|}{ Sexo } \\
\hline Masculino & 28357 & 47,1 & 4408 & 15,5 & $14,8-16,4$ \\
\hline Feminino & 31845 & 52,9 & 6711 & 21,1 & $20,2-21,9$ \\
\hline \multicolumn{6}{|l|}{ Idade } \\
\hline $18-49$ & 39594 & 65,8 & 5517 & 13,9 & $13,3-14,6$ \\
\hline $50-59$ & 9742 & 16,2 & 2548 & 26,2 & $24,6-27,8$ \\
\hline 60 e mais & 10866 & 18,0 & 3053 & 28,1 & $26,6-29,7$ \\
\hline \multicolumn{6}{|l|}{ Sócioeconômicos } \\
\hline \multicolumn{6}{|l|}{ Escolaridade } \\
\hline Sem escolaridade & 8240 & 13,7 & 2109 & 25,6 & $23,9-27,4$ \\
\hline Ensino Fundamental Incompleto & 15198 & 25,2 & 3651 & 24,0 & $22,8-25,3$ \\
\hline Ensino Fundamental Completo & 9347 & 15,5 & 1476 & 15,8 & $14,5-17,2$ \\
\hline Ensino Medio Completo & 16878 & 28,0 & 2405 & 14,2 & $13,3-15,2$ \\
\hline Superior ou mais & 10539 & 17,5 & 1478 & 14,0 & $12,8-15,3$ \\
\hline \multicolumn{6}{|l|}{ Raça/Cor } \\
\hline Não Branco & 31629 & 52,5 & 5613 & 17,7 & $17,0-18,6$ \\
\hline Branco & 28573 & 47,5 & 5505 & 19,3 & $18,4-20,2$ \\
\hline \multicolumn{6}{|l|}{ Estrato Social } \\
\hline $\mathrm{D} / \mathrm{E}$ & 14085 & 23,4 & 3106 & 22,1 & $20,7-23,4$ \\
\hline $\mathrm{C}$ & 25738 & 42,8 & 4787 & 18,6 & $17,8-19,5$ \\
\hline $\mathrm{A} / \mathrm{B}$ & 20379 & 33,9 & 3226 & 15,8 & $14,8-16,9$ \\
\hline
\end{tabular}

\section{Condições de Saúde}

Autoavaliação de saúde

Ruim ou Muito Ruim

Regular

Boa

Muito Boa

Depressão

Não

Sim

Comorbidades

Nenhuma
1 doença
2 doenças
3 ou mais doenças

Acesso a serviços de saúde

Domicílio coberto pela ESF

\begin{tabular}{|c|c|c|c|c|c|}
\hline Ruim ou Muito Ruim & 3506 & 5,8 & 1540 & 43,9 & $41,1-46,8$ \\
\hline Regular & 16887 & 28,0 & 4790 & 28,4 & $27,1-29,7$ \\
\hline Boa & 31801 & 52,8 & 4114 & 12,9 & $12,3-13,7$ \\
\hline Muito Boa & 8008 & 13,3 & 674 & 8,4 & $7,4-9,6$ \\
\hline \multicolumn{6}{|l|}{ essão } \\
\hline Não & 46292 & 76,9 & 9310 & 16,7 & $16,1-17,4$ \\
\hline Sim & 13910 & 23,1 & 1808 & 39,3 & $36,9-41,8$ \\
\hline \multicolumn{6}{|l|}{ prbidades } \\
\hline Nenhuma & 39045 & 64,9 & 5024 & 12,9 & $12,2-13,5$ \\
\hline 1 doença & 14082 & 23,4 & 3464 & 24,6 & $23,3-25,9$ \\
\hline 2 doenças & 5052 & 8,4 & 1685 & 33,4 & $30,9-35,9$ \\
\hline 3 ou mais doenças & 2023 & 3,4 & 946 & 46,8 & $42,9-50,6$ \\
\hline \multicolumn{6}{|l|}{ o a serviços de saúde } \\
\hline \multicolumn{6}{|l|}{ cílio coberto pela ESF } \\
\hline Sim & 32875 & 54,6 & 6477 & 19,7 & $18,8-20,6$ \\
\hline Não & 20826 & 34,6 & 3515 & 16,9 & $15,9-17,9$ \\
\hline Não sei & 6502 & 10,8 & 1126 & 17,3 & $15,9-18,9$ \\
\hline \multicolumn{6}{|l|}{ de Saúde } \\
\hline Sim & 18217 & 30,3 & 3316 & 18,2 & $17,1-19,4$ \\
\hline Não & 41985 & 69,7 & 7803 & 18,6 & $17,9-19,3$ \\
\hline
\end{tabular}


Tabela 1. Características da população de 18 anos e mais, prevalência e distribuição do PCC segundo caracteristicas demográficas, socioeconômicas, condições de saúde, acesso a serviços de saúde, contextuais e grau de limitações. Brasil, 2013.

\begin{tabular}{lccccc} 
& \multicolumn{2}{c}{ Amostra } & \multicolumn{3}{c}{ PCC } \\
\cline { 2 - 6 } & $\mathbf{n}$ & $\%$ & $\mathbf{n}$ & $\begin{array}{c}\text { Prevalência } \\
\text { PCC }\end{array}$ & IC 95\% \\
\hline $\begin{array}{l}\text { Regionais } \\
\text { Regiões }\end{array}$ & & & & & \\
$\quad$ Norte & 4479 & 7,4 & 755 & 16,9 & $15,3-18,6$ \\
$\quad$ Nordeste & 16026 & 26,6 & 3071 & 19,2 & $18,1-20,3$ \\
$\quad$ Sudeste & 26365 & 43,8 & 4469 & 16,9 & $15,9-18,0$ \\
$\quad$ Sul & 8898 & 14,8 & 2076 & 23,3 & $21,6-25,2$ \\
$\quad$ Centro-oeste & 4434 & 7,4 & 748 & 16,9 & $15,7-18,1$ \\
Local do domicílio & & & & & \\
$\quad$ Rural & 8302 & 13,8 & 1771 & 21,3 & $19,6-23,1$ \\
$\quad$ Urbano & 51900 & 86,2 & 9348 & 18,0 & $17,3-18,7$ \\
\hline
\end{tabular}

Fonte: Pesquisa Nacional de Saúde (PNS), 2013.

Tabela 2. Proporção da utilização dos tipos de tratamento para PCC e razão de chance (odds) de realização de algum tratamento, segundo caracteristicas demográficas, socioeconômicas, condições de saúde, acesso a serviços de saúde e contextuais . Brasil, 2013.

\begin{tabular}{|c|c|c|c|c|c|c|c|c|}
\hline & & \multicolumn{5}{|c|}{ Tipos de tratamento } & \multicolumn{2}{|c|}{$\begin{array}{c}\text { Chance de } \\
\text { realização de } \\
\text { algum tratamento }\end{array}$} \\
\hline & & $\begin{array}{l}\text { Não realiza } \\
\text { tratamento }\end{array}$ & $\begin{array}{c}\text { Somente } \\
\text { Medicamento }\end{array}$ & $\begin{array}{c}\text { Somente } \\
\text { Fisioterapia/ } \\
\text { Exercícios }\end{array}$ & $\begin{array}{c}\text { Medicamento } \\
\text { e Fisioterapia/ } \\
\text { exercícios }\end{array}$ & $\begin{array}{c}\text { Somente } \\
\text { acupuntura } \\
\text { ou outros }\end{array}$ & $\begin{array}{c}\text { OR } \\
\text { Bruta }\end{array}$ & IC \\
\hline Total & & 46,4 & 31,6 & 10,4 & 8,4 & 3,2 & - & - \\
\hline \multicolumn{9}{|c|}{ Demográficos } \\
\hline \multicolumn{9}{|l|}{ Sexo } \\
\hline & Masculino & 50,6 & 30,6 & 9,2 & 5,9 & 3,7 & 1 & - \\
\hline & Feminino & 43,6 & 32,2 & 11,2 & 10,1 & 2,9 & 1,32 & $1,16-1,51$ \\
\hline \multicolumn{9}{|c|}{ Idade } \\
\hline & $18-49$ & 49,4 & 30,2 & 9,8 & 7,4 & 3,2 & 1 & - \\
\hline & $50-59$ & 42,9 & 33,0 & 10,7 & 10,3 & 3,2 & 1,30 & $1,10-1,53$ \\
\hline & 60 e mais & 43,9 & 32,8 & 11,3 & 8,7 & 3,4 & 1,25 & $1,08-1,45$ \\
\hline \multicolumn{9}{|c|}{ Sócioeconômicos } \\
\hline \multicolumn{9}{|c|}{ Escolaridade } \\
\hline & $\begin{array}{l}\text { Sem } \\
\text { escolaridade }\end{array}$ & 47,2 & 40,0 & 4,5 & 6,5 & 1,8 & 1 & - \\
\hline & $\begin{array}{l}\text { Ensino } \\
\text { Fundamental } \\
\text { Incompleto }\end{array}$ & 44,8 & 35,7 & 7,9 & 8,1 & 3,5 & 1,11 & $0,93-1,31$ \\
\hline & $\begin{array}{l}\text { Ensino } \\
\text { Fundamental } \\
\text { Completo }\end{array}$ & 49,4 & 29,2 & 9,5 & 9,3 & 2,6 & 0,92 & $0,73-1,15$ \\
\hline & $\begin{array}{l}\text { Ensino Medio } \\
\text { Completo }\end{array}$ & 49,8 & 26,1 & 12,1 & 8,8 & 3,1 & 0,90 & $0,74-1,10$ \\
\hline & $\begin{array}{l}\text { Superior ou } \\
\text { mais }\end{array}$ & 40,5 & 20,5 & 23,1 & 10,6 & 5,3 & 1,31 & $1,05-1,64$ \\
\hline
\end{tabular}


Tabela 2. Proporção da utilização dos tipos de tratamento para PCC e razão de chance (odds) de realização de algum tratamento, segundo caracteristicas demográficas, socioeconômicas, condições de saúde, acesso a serviços de saúde e contextuais . Brasil, 2013.

\begin{tabular}{|c|c|c|c|c|c|c|c|}
\hline & \multicolumn{5}{|c|}{ Tipos de tratamento } & \multicolumn{2}{|c|}{$\begin{array}{c}\text { Chance de } \\
\text { realização de } \\
\text { algum tratamento }\end{array}$} \\
\hline & $\begin{array}{l}\text { Não realiza } \\
\text { tratamento }\end{array}$ & $\begin{array}{c}\text { Somente } \\
\text { Medicamento }\end{array}$ & $\begin{array}{c}\text { Somente } \\
\text { Fisioterapia/ } \\
\text { Exercícios }\end{array}$ & $\begin{array}{c}\text { Medicamento } \\
\text { e Fisioterapia/ } \\
\text { exercícios }\end{array}$ & $\begin{array}{c}\text { Somente } \\
\text { acupuntura } \\
\text { ou outros }\end{array}$ & $\begin{array}{c}\text { OR } \\
\text { Bruta }\end{array}$ & IC \\
\hline \multicolumn{8}{|l|}{ Raça/Cor } \\
\hline Não Branco & 48,0 & 33,5 & 8,3 & 7,5 & 2,7 & 1 & - \\
\hline Branco & 44,7 & 29,5 & 12,6 & 9,4 & 3,8 & 0,87 & $0,76-1,00$ \\
\hline \multicolumn{8}{|l|}{ Estrato Social } \\
\hline $\mathrm{D} / \mathrm{E}$ & 48,4 & 38,5 & 4,5 & 5,7 & 2,8 & 1 & - \\
\hline $\mathrm{C}$ & 47,3 & 32,6 & 9,3 & 8,2 & 2,7 & 1,05 & $0,90-1,22$ \\
\hline $\mathrm{A} / \mathrm{B}$ & 43,1 & 23,3 & 17,8 & 11,4 & 4,4 & 1,24 & $1,05-1,47$ \\
\hline \multicolumn{8}{|l|}{ Condições de Saúde } \\
\hline \multicolumn{8}{|l|}{ Autoavaliação de saúde } \\
\hline $\begin{array}{l}\text { Ruim ou } \\
\text { Muito Ruim }\end{array}$ & 39,3 & 43,4 & 5,4 & 9,7 & 2,1 & 1 & - \\
\hline Regular & 43,2 & 35,5 & 8,7 & 9,3 & 3,3 & 0,85 & $0,70-1,03$ \\
\hline Boa & 51,7 & 24,6 & 12,6 & 7,7 & 3,4 & 0,61 & $0,50-0,73$ \\
\hline Muito Boa & 52,2 & 19,0 & 20,4 & 4,0 & 4,5 & 0,60 & $0,44-0,81$ \\
\hline \multicolumn{8}{|l|}{ Depressão } \\
\hline Não & 47,8 & 30,9 & 10,5 & 7,6 & 3,1 & 1 & - \\
\hline Sim & 39,0 & 34,8 & 9,9 & 12,7 & 3,6 & 1,44 & $1,21-1,71$ \\
\hline \multicolumn{8}{|l|}{ Comorbidades } \\
\hline Nenhuma & 50,4 & 29,3 & 9,7 & 7,3 & 3,3 & 1 & - \\
\hline 1 doença & 46,8 & 31,3 & 10,9 & 8,0 & 3,0 & 1,15 & $0,99-1,35$ \\
\hline 2 doenças & 39,5 & 34,8 & 12,0 & 10,4 & 3,2 & 1,56 & $1,30-1,86$ \\
\hline $\begin{array}{l}3 \text { ou mais } \\
\text { doenças }\end{array}$ & 35,7 & 38,9 & 9,6 & 12,5 & 3,3 & 1,83 & $1,42-2,37$ \\
\hline \multicolumn{8}{|c|}{ Intensidade da limitação por PCC } \\
\hline Sem limitação & 57,6 & 20,7 & 13,0 & 4,5 & 4,3 & 1 & - \\
\hline $\begin{array}{l}\text { Pouca } \\
\text { limitação }\end{array}$ & 45,5 & 31,5 & 11,4 & 8,5 & 3,1 & 1,63 & $1,37-1,93$ \\
\hline $\begin{array}{l}\text { Moderada } \\
\text { limitação }\end{array}$ & 40,8 & 36,9 & 8,4 & 11,4 & 2,6 & 1,97 & $1,62-2,39$ \\
\hline $\begin{array}{l}\text { Intensa ou } \\
\text { Muito intensa } \\
\text { limitação }\end{array}$ & 32,1 & 47,4 & 5,6 & 12,9 & 2,0 & 2,87 & $2,32-3,55$ \\
\hline
\end{tabular}

entre pessoas com nível superior de escolaridade $(5,3 \%)$ e de estrato social A e B $(4,4 \%)$. Ser de cor branca também tem impacto positivo.

A melhor condição de saúde impacta positivamente na realização de acupuntura e outras práticas, sendo esta superior entre os com autoavaliação da saúde muito boa $(4,5 \%)$ e sem limitações causadas pelo PCC (4,3\%). O maior acesso a plano de saúde teve impacto positivo na realização dessas práticas $(4,5 \%)$ se comparado às pes- 
Tabela 2. Proporção da utilização dos tipos de tratamento para PCC e razão de chance (odds) de realização de algum tratamento, segundo caracteristicas demográficas, socioeconômicas, condições de saúde, acesso a serviços de saúde e contextuais . Brasil, 2013.

\begin{tabular}{|c|c|c|c|c|c|c|c|}
\hline & \multicolumn{5}{|c|}{ Tipos de tratamento } & \multicolumn{2}{|c|}{$\begin{array}{c}\text { Chance de } \\
\text { realização de } \\
\text { algum tratamento }\end{array}$} \\
\hline & $\begin{array}{l}\text { Não realiza } \\
\text { tratamento }\end{array}$ & $\begin{array}{c}\text { Somente } \\
\text { Medicamento }\end{array}$ & $\begin{array}{c}\text { Somente } \\
\text { Fisioterapia/ } \\
\text { Exercícios } \\
\end{array}$ & $\begin{array}{c}\text { Medicamento } \\
\text { e Fisioterapia/ } \\
\text { exercícios }\end{array}$ & $\begin{array}{c}\text { Somente } \\
\text { acupuntura } \\
\text { ou outros }\end{array}$ & $\begin{array}{c}\text { OR } \\
\text { Bruta }\end{array}$ & IC \\
\hline \multicolumn{8}{|l|}{ Acesso a serviços de saúde } \\
\hline \multicolumn{8}{|l|}{ Domicílio coberto pela ESF } \\
\hline Sim & 45,8 & 34,1 & 8,6 & 9,0 & 2,5 & 1 & - \\
\hline Não & 46,9 & 27,6 & 13,2 & 8,1 & 4,2 & 0,96 & $0,83-1,10$ \\
\hline Não sei & 48,1 & 29,3 & 12,3 & 6,2 & 4,1 & 0,91 & $0,74-1,13$ \\
\hline \multicolumn{8}{|l|}{ Plano de Saúde } \\
\hline Sim & 43,8 & 23,4 & 17,9 & 10,5 & 4,5 & 1 & - \\
\hline Não & 47,5 & 35,0 & 7,2 & 7,6 & 2,7 & 1,16 & $1,00-1,35$ \\
\hline \multicolumn{8}{|l|}{ Regionais } \\
\hline \multicolumn{8}{|l|}{ Regiões } \\
\hline Norte & 45,9 & 34,2 & 9,1 & 6,2 & 4,5 & 1 & - \\
\hline Nordeste & 49,0 & 35,8 & 6,7 & 5,9 & 2,5 & 0,88 & $0,72-1,08$ \\
\hline Sudeste & 48,0 & 27,7 & 12,0 & 8,8 & 3,5 & 0,92 & $0,75-1,13$ \\
\hline Sul & 41,6 & 31,5 & 12,5 & 11,0 & 3,4 & 1,19 & $0,95-1,49$ \\
\hline Centro-oeste & 39,7 & 34,5 & 11,5 & 11,6 & 2,6 & 1,29 & $1,01-1,64$ \\
\hline \multicolumn{8}{|l|}{ Local do domicílio } \\
\hline Rural & 48,0 & 39,0 & 5,0 & 4,9 & 3,2 & 1 & - \\
\hline Urbano & 46,1 & 30,1 & 11,4 & 9,1 & 3,2 & 1,08 & $0,94-1,25$ \\
\hline
\end{tabular}

Fonte: Pesquisa Nacional de Saúde (PNS), 2013.

de tratamento do que homens (OR 1,32 IC95\% 1,16-1,51). Em geral, pessoas a partir de 50 anos têm mais chances de realizar algum tratamento do que os mais jovens. Adultos entre 50 e 59 anos apresentaram $30 \%$ mais chances de realizar algum tratamento em relação aos mais jovens (OR 1,30 IC 1,10-1,53) e idosos apresentaram 25\% mais chances (OR 1,25 IC95\%1,08 -1,45). Melhores condições socioeconômicas refletiram maior chance de realização de algum tratamento sendo $31 \%$ mais chances para pessoas com ensino superior em relação aos sem escolaridade (OR 1,31 IC95\% 1,05 - 1,64) e $24 \%$ mais chances entre os de estrato social A/B em relação aos de D/E (OR 1,24 IC95\%1,05 - 1,47).

Piores condições de saúde aumentam a chance de realização de algum tratamento. A intensidade da limitação foi a variável com correlação mais significativa para realização de tratamento. Quanto maior sua intensidade, maior é a chance de realização de tratamento, chegando a quase três vezes mais chances para pessoas com limitação intensa a muito intensa (OR 2,87 IC95\%2,32
- 3,55). Variáveis de acesso a serviços e regionais não apresentaram correlação com a realização ou não de algum tratamento para o PCC. Nos modelos seguintes apresenta-se a razão de chance de realização de fisioterapia/exercícios e utilização de medicamentos para tratamento do PCC segundo características da população.

Foram observadas diferenças significativas na utilização dos dois principais tipos de tratamento (Tabela 3). Mulheres apresentaram $27 \%$ mais chances de utilização de medicamentos (OR 1,27 IC95\% $1,11-1,45)$ quase duas vezes mais chance de realização de fisioterapia/exercícios como intervenção para o PCC do que homens (OR 1,95 IC95\%1,66 - 2,30) no modelo bruto. Essa relação se mantém no modelo ajustado, no entanto perde força. No modelo ajustado, mulheres apresentaram $20 \%$ mais chances de utilização de medicamentos (OR1,20 IC95\%1,04 - 1,38) e 37\% mais chances de realização de fisioterapia (OR 1,37 IC95\%1,13 - 1,66), evidenciando, ainda assim, uma diferença maior entre os sexos para realização desta última intervenção. 
Tabela 3. Razão de chance (odds) de uso de medicamento e de uso de fisioterapia para tratamento de PCC segundo características demográficas, socioeconômicas, condições de saúde, acesso a serviços de saúde e contextuais. Brasil, 2013.

\begin{tabular}{|c|c|c|c|c|c|c|c|}
\hline \multicolumn{4}{|c|}{ Uso de medicamento } & \multicolumn{4}{|c|}{ Uso de fisioterapia } \\
\hline $\begin{array}{c}\text { OR } \\
\text { bruta }\end{array}$ & IC 95\% & $\begin{array}{c}\text { OR } \\
\text { ajustada }\end{array}$ & IC $95 \%$ & $\begin{array}{c}\text { OR } \\
\text { bruta }\end{array}$ & IC 95\% & $\begin{array}{c}\text { OR } \\
\text { ajustada }\end{array}$ & IC $95 \%$ \\
\hline
\end{tabular}

\section{Demográficos}

Sexo

\begin{tabular}{|c|c|c|c|c|c|c|c|c|}
\hline Homens & 1 & - & 1,00 & & 1 & - & 1,00 & \\
\hline Mulheres & 1,27 & $1,11-1,45$ & 1,20 & $1,04-1,38$ & 1,95 & $1,66-2,30$ & 1,37 & $1,13-1,66$ \\
\hline
\end{tabular}

Idade

\begin{tabular}{|c|c|c|c|c|c|c|c|c|}
\hline $18-49$ & 1 & - & 1,00 & & 1 & - & 1,00 & - \\
\hline $50-59$ & 1,27 & $1,07-1,49$ & 1,01 & $0,84-1,21$ & 2,36 & $1,91-2,91$ & 1,38 & $1,09-1,74$ \\
\hline 60 e mais & 1,18 & $1,00-1,38$ & 0,89 & $0,73-1,08$ & 2,42 & $2,05-2,86$ & 1,42 & $1,13-1,80$ \\
\hline
\end{tabular}

Sócioeconômicos

Escolaridade

\begin{tabular}{|c|c|c|c|c|c|c|c|c|}
\hline $\begin{array}{l}\text { Sem } \\
\text { escolaridade }\end{array}$ & 1 & - & 1,00 & & 1 & - & 1,00 & - \\
\hline $\begin{array}{l}\text { Fundamental } \\
\text { incompleto }\end{array}$ & 0,90 & $0,76-1,07$ & 0,91 & $0,75-1,10$ & 1,38 & $1,05-1,82$ & 1,26 & $0,95-1,68$ \\
\hline $\begin{array}{l}\text { Fundamental } \\
\text { completo }\end{array}$ & 0,72 & $0,57-0,91$ & 0,81 & $0,62-1,04$ & 1,05 & $0,78-1,43$ & 1,47 & $1,05-2,07$ \\
\hline $\begin{array}{l}\text { Medio } \\
\text { completo }\end{array}$ & 0,62 & $0,51-0,75$ & 0,76 & $0,59-0,98$ & 1,06 & $0,80-1,41$ & 1,49 & $1,05-2,11$ \\
\hline $\begin{array}{l}\text { Superior ou } \\
\text { mais }\end{array}$ & 0,52 & $0,41-0,66$ & 0,74 & $0,55-1,01$ & 1,71 & $1,29-2,27$ & 2,39 & $1,63-3,49$ \\
\hline
\end{tabular}

Raça/Cor

\begin{tabular}{|c|c|c|c|c|c|c|c|c|}
\hline Não Branco & 1 & - & 1,00 & & 1 & - & 1,00 & - \\
\hline Branco & 1,09 & $0,96-1,24$ & 1,04 & $0,89-1,21$ & 1,54 & $1,31-1,81$ & 0,98 & $0,80-1,20$ \\
\hline
\end{tabular}

Estrato Social

$\mathrm{D} / \mathrm{E}$

C

$1 \quad-\quad 1,00$

$\mathrm{A} / \mathrm{B}$

$0,87 \quad 0,75-1,01$

1,00

-

Condições de Saúde

$0,67 \quad 0,57-0,80 \quad 1,22$

$0,94-1,34 \quad 1,45 \quad 1,19-1,78$

1,00

1,43

$1,14-1,79$

$0,94-1,57$

2,10

$1,70-2,58$

1,96

$1,43-2,69$

Autoavaliação de saúde

Ruim ou

Muito Ruim

Regular

$1 \quad-\quad 1,00$

1,00

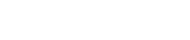

1

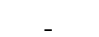

1,00

$0,72 \quad 0,60-0,86 \quad 0,98$

$0,80-1,20$

0,76

$0,58-0,99$

1,11

Boa

$0,42 \quad 0,35-0,51$

0,74

$0,58-0,93$

$0,38 \quad 0,29-0,50$

1,08

$0,81-1,51$

Muito Boa

$0,26 \quad 0,19-0,37 \quad 0,55$

$0,38-0,80$

$0,290,20-0,44$

1,25

$0,78-1,51$

Depressão

$\begin{array}{lcccccccc}\text { Não } & 1 & - & 1,00 & & 1 & - & 1,00 & - \\ \text { Sim } & 1,44 & 1,22-1,71 & 1,11 & 0,90-1,38 & 3,11 & 2,58-3,76 & 0,98 & 0,76-1,27\end{array}$

Comorbidades

$\begin{array}{lcccccccc}\text { Nenhuma } & 1 & - & 1,00 & & 1 & - & 1,00 & - \\ 1 \text { doença } & 1,12 & 0,95-1,33 & 0,99 & 0,82-1,20 & 2,19 & 1,82-2,63 & 0,99 & 0,80-1,23 \\ 2 \text { doenças } & 1,43 & 1,19-1,72 & 1,10 & 0,88-1,38 & 3,62 & 2,95-4,45 & 1,20 & 0,92-1,56 \\ \begin{array}{l}3 \text { ou mais } \\ \text { doenças }\end{array} & 1,83 & 1,44-2,34 & 1,18 & 0,86-1,62 & 5,16 & 3,84-6,95 & 1,04 & 0,70-1,54 \\ \end{array}$


Tabela 3. Razão de chance (odds) de uso de medicamento e de uso de fisioterapia para tratamento de PCC segundo características demográficas, socioeconômicas, condições de saúde, acesso a serviços de saúde e contextuais. Brasil, 2013.

\begin{tabular}{|c|c|c|c|c|c|c|c|c|}
\hline & \multicolumn{4}{|c|}{ Uso de medicamento } & \multicolumn{4}{|c|}{ Uso de fisioterapia } \\
\hline & $\begin{array}{c}\text { OR } \\
\text { bruta }\end{array}$ & IC 95\% & $\begin{array}{c}\text { OR } \\
\text { ajustada }\end{array}$ & IC 95\% & $\begin{array}{c}\text { OR } \\
\text { bruta }\end{array}$ & IC 95\% & $\begin{array}{c}\mathrm{OR} \\
\text { ajustada }\end{array}$ & IC $95 \%$ \\
\hline \multicolumn{9}{|c|}{ Intensidade da limitação por PCC } \\
\hline $\begin{array}{l}\text { Sem } \\
\text { limitação }\end{array}$ & 1 & - & 1,00 & & 1 & - & 1,00 & - \\
\hline $\begin{array}{l}\text { Pouca } \\
\text { limitação }\end{array}$ & 1,98 & $1,67-2,35$ & 1,78 & $1,49-2,12$ & 1,18 & $0,95-1,47$ & 1,44 & $1,14-1,81$ \\
\hline $\begin{array}{l}\text { Moderada } \\
\text { Limitação }\end{array}$ & 2,77 & $2,28-3,37$ & 2,35 & $1,91-2,89$ & 1,17 & $0,90-1,52$ & 1,47 & $1,12-1,93$ \\
\hline $\begin{array}{l}\text { Intensa } \\
\text { ou muito } \\
\text { intensa } \\
\text { limitação }\end{array}$ & 4,52 & $3,63-5,63$ & 3,53 & $2,78-4,48$ & 1,08 & $0,84-1,37$ & 1,48 & $1,14-1,93$ \\
\hline \multicolumn{9}{|l|}{ Acesso a serviços de saúde } \\
\hline \multicolumn{9}{|l|}{ Domicílio coberto pela ESF } \\
\hline $\operatorname{Sim}$ & 1 & - & 1,00 & & 1 & - & 1,00 & - \\
\hline Não & 0,73 & $0,64-0,85$ & 0,88 & $0,75-1,02$ & 1,05 & $0,88-1,24$ & 0,86 & $0,70-1,06$ \\
\hline Não Sei & 0,73 & $0,59-0,90$ & 0,78 & $0,62-0,97$ & 0,92 & $0,69-1,22$ & 0,95 & $0,68-1,32$ \\
\hline \multicolumn{9}{|l|}{ Plano de Saúde } \\
\hline Não & 1 & - & 1,00 & & 1 & - & 1,00 & - \\
\hline Sim & 0,69 & $0,59-0,80$ & 0,85 & $0,71-1,03$ & 1,93 & $1,63-2,27$ & 1,44 & $1,15-1,80$ \\
\hline \multicolumn{9}{|l|}{ Regionais } \\
\hline \multicolumn{9}{|l|}{ Região } \\
\hline Norte & 1 & - & 1,00 & & 1 & - & 1,00 & - \\
\hline Nordeste & 1,06 & $0,86-1,30$ & 0,95 & $0,76-1,17$ & 0,93 & $0,72-1,20$ & 0,80 & $0,62-1,03$ \\
\hline Sudeste & 0,85 & $0,68-1,05$ & 0,86 & $0,68-1,08$ & 1,38 & $1,07-1,78$ & 0,99 & $0,76-1,30$ \\
\hline Sul & 1,09 & $0,87-1,36$ & 0,98 & $0,76-1,27$ & 2,18 & $1,64-2,89$ & 1,27 & $0,93-1,74$ \\
\hline Centro oeste & 1,26 & $0,99-1,61$ & 1,16 & $0,90-1,50$ & 1,52 & $1,18-1,97$ & 1,20 & $0,92-1,56$ \\
\hline \multicolumn{9}{|l|}{ Local do domicílio } \\
\hline Rural & 1 & - & 1,00 & & 1 & - & 1,00 & - \\
\hline Urbano & 0,83 & $0,72-0,96$ & 1,02 & $0,87-1,20$ & 1,80 & $1,44-2,24$ & 1,46 & $1,13-1,87$ \\
\hline
\end{tabular}

Fonte: Pesquisa Nacional de Saúde (PNS), 2013.

O avanço da idade mostrou aumento da chance de realização de fisioterapia/exercícios quando comparado com o grupo mais jovem, chegando a ter duas vezes mais chances de utilizar fisioterapia/exercícios como intervenção entre a população idosa (2,24 IC95\%2,05 - 2,86), na análise bruta. A idade não foi associada à utilização de medicamentos no modelo ajustado, no entanto, aumentou progressivamente às chances de realização de fisioterapia. Indivíduos com 60 anos e mais apresentaram $42 \%$ mais chances de realizar esse tratamento (OR 1,42 IC95\%1,13 1,80) (Tabela 4).

A desigualdade socioeconômica afeta mais a utilização de fisioterapia/exercícios do que uso de medicamentos. Ter ensino superior aumenta 1,7 vezes as chances de realizar fisioterapia/exercícios (IC95\%1,29 - 2,27), na análise bruta. A associação se intensifica quando as demais variáveis são agregadas, aumentando progressivamente e chegando a ser 2,39 vezes maior para individuos com ensino superior (OR 2,39 IC95\%1,63 - 3,49). Por outro lado, a escolaridade não se mostrou associada à utilização de medicamentos.

$\mathrm{O}$ estrato social mostrou forte associação mesmo após ajuste por todas as variáveis. Indivíduos de estratos superiores (A/B) apresentam quase 2 vezes mais chances de realizar fisioterapia (OR 1,96 IC95\%1,43 - 2,69), o que não foi observado quanto à utilização de medicamentos. A cor 
Tabela 4. Razão de chance (odds) de fazer fisioterapia/exercícios para tratamento de PCC segundo características demográficas, socioeconômicas, condições de saúde, acesso a serviços de saúde e contextuais. Brasil, 2013.

\begin{tabular}{|c|c|c|c|c|c|c|c|c|c|c|}
\hline & \multicolumn{2}{|c|}{ Or Bruta } & \multicolumn{2}{|c|}{ Modelo 1} & \multicolumn{2}{|c|}{ Modelo 2} & \multicolumn{2}{|c|}{ Modelo 3} & \multicolumn{2}{|c|}{ Modelo 4} \\
\hline & OR & IC 95\% & OR & IC 95\% & OR & IC 95\% & OR & IC 95\% & OR & IC 95\% \\
\hline \multicolumn{11}{|l|}{ Demográficos } \\
\hline \multicolumn{11}{|l|}{ Sexo } \\
\hline Homens & 1 & - & 1,00 & & 1,00 & - & 1,00 & & 1,00 & \\
\hline Mulheres & 1,95 & $1,66-2,30$ & 1,93 & $1,63-2,28$ & 1,38 & $1,14-1,67$ & 1,38 & $1,14-1,66$ & 1,37 & $1,13-1,66$ \\
\hline \multicolumn{11}{|l|}{ Idade } \\
\hline $18-49$ & 1 & - & 1,00 & & 1,00 & - & 1,00 & - & 1,00 & - \\
\hline $50-59$ & 2,36 & $1,91-2,91$ & 2,37 & $1,92-2,93$ & 1,42 & $1,12-1,79$ & 1,38 & $1,09-1,75$ & 1,38 & $1,09-1,74$ \\
\hline 60 e mais & 2,42 & $2,05-2,86$ & 2,60 & $2,16-3,12$ & 1,50 & $1,20-1,89$ & 1,43 & $1,13-1,81$ & 1,42 & $1,13-1,80$ \\
\hline \multicolumn{11}{|l|}{ Sócioeconômicos } \\
\hline \multicolumn{11}{|l|}{ Escolaridade } \\
\hline Sem escolaridade & 1 & - & 1,00 & - & 1,00 & - & 1,00 & - & 1,00 & - \\
\hline $\begin{array}{l}\text { Fundamental } \\
\text { incompleto }\end{array}$ & 1,38 & $1,05-1,82$ & 1,34 & $1,02-1,77$ & 1,34 & $1,01-1,77$ & 1,31 & $0,99-1,74$ & 1,26 & $0,95-1,68$ \\
\hline $\begin{array}{l}\text { Fundamental } \\
\text { Completo }\end{array}$ & 1,05 & $0,78-1,43$ & 1,18 & $0,85-1,63$ & 1,60 & $1,14-2,25$ & 1,55 & $1,10-2,17$ & 1,47 & $1,05-2,07$ \\
\hline Médio Completo & 1,06 & $0,80-1,41$ & 1,03 & $0,74-1,43$ & 1,58 & $1,12-2,22$ & 1,50 & $1,06-2,12$ & 1,49 & $1,05-2,11$ \\
\hline Superior ou mais & 1,71 & $1,29-2,27$ & 1,35 & $0,95-1,92$ & 2,63 & $1,81-3,81$ & 2,38 & $1,63-3,47$ & 2,39 & $1,63-3,49$ \\
\hline \multicolumn{11}{|l|}{ Raça/Cor } \\
\hline Não Branco & 1 & - & 1,00 & - & & - & 1,00 & - & 1,00 & - \\
\hline Branco & 1,54 & $1,31-1,81$ & 1,20 & $1,01-1,43$ & 1,08 & $0,90-1,30$ & 1,06 & $0,88-1,28$ & 0,98 & $0,80-1,20$ \\
\hline \multicolumn{11}{|l|}{ Estrato Social } \\
\hline $\mathrm{D} / \mathrm{E}$ & 1 & - & 1,00 & - & 1,00 & - & 1,00 & - & 1,00 & - \\
\hline $\mathrm{C}$ & 1,45 & $1,19-1,78$ & 1,59 & $1,29-1,97$ & 1,64 & $1,32-2,05$ & 1,58 & $1,26-1,98$ & 1,43 & $1,14-1,79$ \\
\hline $\mathrm{A} / \mathrm{B}$ & 2,10 & $1,70-2,58$ & 2,26 & $1,70-2,99$ & 2,48 & $1,83-3,37$ & 2,24 & $1,63-3,07$ & 1,96 & $1,43-2,69$ \\
\hline \multicolumn{11}{|l|}{$\begin{array}{l}\text { Condições de } \\
\text { Saúde }\end{array}$} \\
\hline \multicolumn{11}{|l|}{$\begin{array}{l}\text { Autoavaliação de } \\
\text { saúde }\end{array}$} \\
\hline $\begin{array}{l}\text { Ruim ou Muito } \\
\text { Ruim }\end{array}$ & 1 & - & - & & 1,00 & - & 1,00 & - & 1,00 & - \\
\hline Regular & 0,76 & $0,58-0,99$ & & & 1,17 & $0,84-1,63$ & 1,13 & $0,83-1,53$ & 1,11 & $0,81-1,51$ \\
\hline Boa & 0,38 & $0,29-0,50$ & & & 1,39 & $0,87-2,22$ & 1,13 & $0,81-1,57$ & 1,08 & $0,78-1,51$ \\
\hline Muito Boa & 0,29 & $0,20-0,44$ & & & 1,14 & $0,84-1,55$ & 1,33 & $0,83-2,15$ & 1,25 & $0,78-2,02$ \\
\hline \multicolumn{11}{|l|}{ Depressão } \\
\hline Não & 1 & - & - & & 1,00 & - & 1,00 & - & 1,00 & - \\
\hline Sim & 3,11 & $2,58-3,76$ & & & 0,99 & $0,77-1,28$ & 1,01 & $0,78-1,30$ & 0,98 & $0,76-1,27$ \\
\hline \multicolumn{11}{|l|}{ Comorbidades } \\
\hline Nenhuma & 1 & - & - & & 1,00 & - & 1,00 & - & 1,00 & - \\
\hline 1 doença & 2,19 & $1,82-2,63$ & & & 1,03 & $0,83-1,28$ & 1,02 & $0,82-1,26$ & 0,99 & $0,80-1,23$ \\
\hline 2 doenças & 3,62 & $2,95-4,45$ & & & 1,26 & $0,97-1,65$ & 1,24 & $0,95-1,61$ & 1,20 & $0,92-1,56$ \\
\hline $\begin{array}{l}3 \text { ou mais } \\
\text { doenças }\end{array}$ & 5,16 & $3,84-6,95$ & & & 1,16 & $0,78-1,71$ & 1,11 & $0,75-1,65$ & 1,04 & $0,70-1,54$ \\
\hline \multicolumn{11}{|c|}{$\begin{array}{l}\text { Intensidade da limitação } \\
\text { por PCC }\end{array}$} \\
\hline Sem limitação & 1 & - & - & & 1,00 & - & 1,00 & - & 1,00 & - \\
\hline Pouca limitação & 1,18 & $0,95-1,47$ & & & 1,42 & $1,13-1,79$ & 1,44 & $1,15-1,82$ & 1,44 & $1,14-1,81$ \\
\hline $\begin{array}{l}\text { Moderada } \\
\text { Limitação }\end{array}$ & 1,17 & $0,90-1,52$ & & & 1,45 & $1,11-1,90$ & 1,46 & $1,11-1,92$ & 1,47 & $1,12-1,93$ \\
\hline $\begin{array}{l}\text { Intensa ou muito } \\
\text { intensa limitação }\end{array}$ & 1,08 & $0,84-1,37$ & & & 1,47 & $1,13-1,92$ & 1,50 & $1,15-1,95$ & 1,48 & $1,14-1,93$ \\
\hline
\end{tabular}


Tabela 4. Razão de chance (odds) de fazer fisioterapia/exercícios para tratamento de PCC segundo características demográficas, socioeconômicas, condições de saúde, acesso a serviços de saúde e contextuais. Brasil, 2013.

\begin{tabular}{|c|c|c|c|c|c|c|c|c|c|c|}
\hline & \multicolumn{2}{|c|}{ Or Bruta } & \multicolumn{2}{|c|}{ Modelo 1} & \multicolumn{2}{|c|}{ Modelo 2} & \multicolumn{2}{|c|}{ Modelo 3} & \multicolumn{2}{|c|}{ Modelo 4} \\
\hline & OR & IC 95\% & OR & IC $95 \%$ & OR & IC $95 \%$ & OR & IC $95 \%$ & OR & IC 95\% \\
\hline \multicolumn{11}{|c|}{$\begin{array}{l}\text { Acesso a serviços de } \\
\text { saúde }\end{array}$} \\
\hline \multicolumn{11}{|c|}{ Domicílio coberto pela ESF } \\
\hline Sim & 1 & - & - & & - & - & 1,00 & - & 1,00 & - \\
\hline Não & 1,05 & $0,88-1,24$ & & & & - & 0,87 & $0,71-1,06$ & 0,86 & $0,70-1,06$ \\
\hline Não Sei & 0,92 & $0,69-1,22$ & & & & - & 0,94 & $0,68-1,31$ & 0,95 & $0,68-1,32$ \\
\hline \multicolumn{11}{|l|}{ Plano de Saúde } \\
\hline Não & 1 & - & - & & - & - & 1,00 & - & 1,00 & - \\
\hline Sim & 1,93 & $1,63-2,27$ & & & & - & 1,48 & $1,18-1,85$ & 1,44 & $1,15-1,80$ \\
\hline \multicolumn{11}{|l|}{ Contextuais } \\
\hline \multicolumn{11}{|l|}{ Região } \\
\hline Norte & 1 & - & - & & - & - & - & & 1,00 & - \\
\hline Nordeste & 0,93 & $0,72-1,20$ & & & & - & & & 0,80 & $0,62-1,03$ \\
\hline Sudeste & 1,38 & $1,07-1,78$ & & & & - & & & 0,99 & $0,76-1,30$ \\
\hline Sul & 2,18 & $1,64-2,89$ & & & & - & & & 1,27 & $0,93-1,74$ \\
\hline Centro oeste & 1,52 & $1,18-1,97$ & & & & - & & & 1,20 & $0,92-1,56$ \\
\hline \multicolumn{11}{|c|}{ Local do domicílio } \\
\hline Rural & 1 & - & - & & - & - & - & & 1,00 & - \\
\hline Urbano & 1,80 & $1,44-2,24$ & & & & - & & & 1,46 & $1,13-1,87$ \\
\hline
\end{tabular}

da pele mostrou associação com a realização de fisioterapia/exercícios no modelo bruto (OR 1,54 IC95\%1,31 - 1,81), no entanto perde significância no modelo ajustado.

Quanto às condições de saúde, autoavaliação, ter depressão e ter comorbidades e intensidade da limitação - não apresentaram associação com a realização de fisioterapia/exercícios, tanto na análise bruta quanto na multivariada. Verificouse que a melhor autoavaliação de saúde, diminui as chances de utilização de medicamentos. Pessoas com autoavaliação de saúde muito boa apresentaram $45 \%$ menos chances de utilizar medicamentos do que quem tem autoavaliação de saúde ruim ou muito ruim (OR 0,55 IC95\%0,38$0,80)$, no modelo ajustado. A maior intensidade da limitação mostrou-se fortemente associada à utilização de medicamentos, aumentando progressivamente as chances de utilização em ambos os modelos. Indivíduos com limitação intensa ou muito intensa chegam a ter 3,5 vezes mais chances de utilizar medicamentos no modelo ajusta- do. Ter depresssão ou comorbidades mostraram associação com uso de medicação na análise bruta, mas perdeu significância no modelo ajustado.

Das variáveis de acesso aos serviços de saúde, residir em domicílio com cobertura da ESF não mostrou associação com a realização fisioterapia/exercícios e sua associação com a utilização de medicamentos perde significância no modelo ajustado. Ter plano de saúde foi associado somente à realização de fisioterapia mesmo após o ajuste por outras variáveis (OR 1,44 IC95\%1,15 $-1,80)$. Ao passo que não houve associação significativa quanto à utilização de medicamento e realização de fisioterapia/exercícios por regiões, pessoas residentes em áreas urbanas apresentaram mais chances de realizar esse tratamento do que em áreas rurais (OR 1,46 IC95\%1,13-1,87). Residir em região urbana foi associado a maior chance de utilização de fisioterapia (OR1,80 IC95\%1,44 - 2,24) no modelo bruto. No modelo ajustado a associação permanece significante (OR 1,46 IC95\%1,13 - 1,87). 


\section{Discussão}

Neste trabalho mostrou-se que, no Brasil, os principais tratamentos utilizados para PCC são a fisioterapia/exercícios e o uso de medicamentos. Quase a metade da população $(46,4 \%)$ com PCC não faz nenhum tipo de tratamento, valor que pode ser considerado alto quando comparado a outros países. Em estudo realizado na Carolina do Norte, verificou-se que cerca de $20 \%$ das pessoas com problemas de coluna não buscavam tratamento ${ }^{23}$.

Embora os sistemas de diagnóstico e tratamento disponíveis na literatura ainda não permitam afirmar que existe um "padrão ouro" para a abordagem do $\mathrm{PCC}^{15}$, a realização de algum tratamento contínuo que permita reduzir a carga desse problema na qualidade vida, evitar cirurgias e reduzir os custos para o sistema de saúde é amplamente indicada na literatura ${ }^{24}$. Pesquisas em diversos países mostraram que a reabilitação envolvendo exercícios, educação e envolvimento ativo do paciente tem se mostrado eficaz em reduzir as limitações e incapacidades provocadas pelo PCC $^{14,15}$.

Apesar dessas recomendações, observou-se neste estudo a baixa procura por tratamentos contínuos, como a realização de fisioterapia/ exercícios, assim como encontrado em estudo internacional de revisão sistemática ${ }^{25}$. Em contrapartida, o uso de medicamentos - associado a fases agudas de dor - foi a principal intervenção utilizada para tratar o PCC, resultado também consistente com a literatura ${ }^{11,26}$. $\mathrm{O}$ uso mais frequente de medicamentos em detrimento de tratamentos contínuos, como a fisioterapia, pode ser explicado pelo seu alto custo e demora em obter resultados ${ }^{27}$.

Verificou-se também nesse estudo que, com o aumento do grau da limitação por PCC elevase significativamente a chance de uso de medicamentos, chegando a ser 3,5 vezes maior entre os que têm limitação intensa ou muito intensa. Embora tenha sido observada uma alta utilização dessa intervenção, na PNS não há informação sobre o tipo de medicamentos utilizados e nem se foram prescritos por um médico. Um estudo estadunidense realizado entre 1999 e 2010 observa que os medicamentos mais utilizados foram narcóticos, benzodiazepinas e relaxantes muscula$\mathrm{res}^{24}$. Uma revisão sistemática sobre problemas de coluna na África mostrou que os medicamentos mais utilizados foram analgésicos ${ }^{16}$.

Embora Krismer e Van Tulder ${ }^{11}$ apontem que há evidências para apoiar o uso de analgésicos simples, anti-inflamatórios não esteroidais e relaxantes musculares para a redução da dor causada por problemas de coluna, Martell et al..$^{28}$, num estudo de metanálise realizado entre 1966 e 2005 , aponta que o uso indiscriminado de opióides é um sério problema para a saúde pública, visto que tais medicamentos são comumente prescritos para tratar PCC devido à sua eficácia para o alívio da dor a curto prazo. Esse resultado reforça a teoria anteriormente citada sobre a menor aderência a tratamentos com ação a médio e longo prazos.

Observou-se neste estudo que a presença de limitações em qualquer intensidade implica maior uso da fisioterapia como tratamento, assim como encontrado em estudo canadense ${ }^{17}$. Apesar disso, a intensidade da limitação não impacta na chance de realização dessa intervenção. Apesar de ser considerada mais cara, a prática de fisioterapia pode reduzir o tempo gasto em hospitais e, portanto, reduzir os custos de tratamento com o problema $^{29}$.

Constatou-se que a escolaridade alta e maior renda está associada com a maior chance de uso de fisioterapia, resultado similar ao encontrado por Freburger em estudo de base populacional realizado na Carolina do Norte $^{30}$, que aponta que indivíduos com maior escolaridade e maior renda são mais propensos a consultar um fisioterapeuta. Num estudo transversal de base populacional realizado em Pelotas/RS $(\mathrm{n}=3.100)^{29}$, o estrato social mais elevado também foi associado à maior realização dessa intervenção, mas o grau de escolaridade não mostrou associação significativa. A associação encontrada neste estudo entre a alta escolaridade e a realização de fisioterapia pode ter sido influenciada pela maior escolaridade entre pessoas de estratos sociais mais elevados.

O tipo de intervenção realizada para o tratamento do PCC está associado à condição socioeconômica do indivíduo, como seu estrato social e renda. Neste estudo, a variável raça não foi um preditor estatisticamente significativo da variável dependente em nenhum dos modelos considerados. Outros estudos também indicam que a procura de cuidados para tratamento do PCC foi semelhante entre as raças ${ }^{31}$. Assim como na literatura, os achados deste estudo mostraram que indivíduos que tinham seguro privado de saúde são mais propensos a consultar um fisioterapeuta ${ }^{30}$. Tal condição também pode ser influenciada pela relação entre ter plano privado de saúde e renda/ estrato social.

Apesar de estudos observarem uma melhora no acesso aos serviços de saúde no Brasil ${ }^{32,33}$, este trabalho mostrou que a utilização de algum 
tipo de tratamento ainda é menor entre populações mais vulneráveis, com menor estrato social. Quanto ao sexo, este estudo mostrou que mulheres são mais propensas a buscarem tratamento, resultado similar ao encontrado por Carey, em estudo representativo da Carolina do $\mathrm{Norte}^{23}$. O mesmo estudo observa que a busca por cuidados é maior entre os que têm mais dores e limitações, assim como detectado neste trabalho.

No Brasil, dados com representatividade populacional sobre o acesso aos serviços de saúde, consultas médicas e tratamentos relacionados ao PCC são escassos. Por ser baixa a frequência de internação por essa causa, não é possível realizar estudos epidemiológicos a partir dos registros de sistemas de informação em Saúde. Inquéritos de Saúde domiciliares têm a vantagem da representatividade populacional e de considerarem não apenas indivíduos que buscaram serviços de saúde. Para estudar as intervenções utilizadas para o tratamento do PCC é fundamental o registro de informações fora do âmbito formal de saúde, já que algumas intervenções informais, como a realização de atividade física, podem ser mais eficazes para enfrentar o problema do que práticas tradicionais, como fisioterapia e quiropraxia ${ }^{34}$. Além disso, práticas comuns como a automedicação, não se encontram em registros hospitalares.

A PNS é o primeiro inquérito em saúde brasileiro com representatividade nacional que considera perguntas sobre os tipos de intervenção utilizados para tratar do PCC, além de tempo com a doença, idade de início e limitações ocasionadas por este problema de saúde. As perguntas sobre tratamento de coluna incluíram tanto as terapêuticas, orientadas por um profissional da saúde, como as de iniciativa do próprio paciente (exercício ou fisioterapia, medicamentos ou injeções, acupuntura e outros). No entanto, deve-se considerar algumas limitações da pesquisa, como a categorização dos tipos de intervenção. A PNS não separa a fisioterapia de exercícios físicos, sendo esta última prática altamente recomendada na literatura para prevenção do PCC.

Quanto à utilização de medicamentos/injeções, não há informações sobre o tipo de medicamento utilizado e nem se este foi prescrito por um médico. Assim, torna-se inviável a realização de estudos sobre automedicação. Outra limitação da pesquisa é que a mesma só capta informações sobre pessoas que estavam realizando tratamento para PCC no momento da entrevista, sem contemplar se já foi feito algum tratamento anteriormente e sua duração. Nesse sentido, não é possível analisar a continuidade dos tratamentos e contrastar com o uso de intervenções pontuais, discussão central no tratamento do PCC.

Estudos apontaram a consulta médica ou busca por algum profissional da saúde como uma das iniciativas mais comuns entre portadores de $\mathrm{PCC}^{23,35,36}$. Os médicos foram os profissionais de saúde mais consultados pelos pacientes, conforme observado em estudo realizado no continente africano ${ }^{16}$. A quiropraxia foi uma prática frequente em estudos internacionais ${ }^{35,37}$, no entanto no Brasil não foi considerada isoladamente.

\section{Conclusão}

A análise da associação entre as variáveis e apresentadas neste trabalho e o tratamento do PCC mostrou que a realização de tratamentos contínuos como fisioterapia foi fortemente associada com a melhor situação socioeconômica. Já o uso de medicamentos não apresentou grandes diferenças segundo essas características. Seu maior uso está associado a piores condições de saúde. 


\section{Colaboradores}

DE Romero, J Muzy: concepção, planejamento, análise, interpretação e redação do trabalho. L Maia, AP Marques: análise, interpretação e redação do trabalho. PRB Souza Júnior: análise e interpretação de dados. D Castanheira: interpretação de dados e redação do trabalho.

\section{Referências}

1. GBD 2016 Disease and Injury Incidence and Prevalence Collaborators. Global, regional, and national incidence, prevalence, and years lived with disability for 328 diseases and injuries for 195 countries, 19902016: a systematic analysis for the Global Burden of Disease Study 2016. Lancet. 2017; 390(10100):12111259.

2. Oliveira MM, Andrade SSCDA, Souza CAV, Ponte JN, Szwarcwald CL, Malta DC. Problema crônico de coluna e diagnóstico de distúrbios osteomusculares relacionados ao trabalho (DORT) autorreferidos no Brasil: Pesquisa Nacional de Saúde, 2013. Epidemiol e Serviços Saúde. 2015; 24(2):287-296.

3. Malta DC, Stopa SR, Szwarcwald CL, Gomes NL, Silva Júnior JB, Reis AAC. A vigilância e o monitoramento das principais doenças crônicas não transmissíveis no Brasil - Pesquisa Nacional de Saúde, 2013. Rev Bras Epidemiol 2015; 18(Supl. 2):3-16.

4. Romero D, Santana D, Borges P, Marques A, Castanheira D, Rodrigues JM, Sabbadini L. Prevalência, fatores associados e limitações relacionados ao problema crônico de coluna entre adultos e idosos no Brasil. Cad Saude Publica 2017; 34(2):e00012817.

5. Meucci RD, Fassa AG, Faria NMX. Prevalence of chronic low back pain: systematic review. Rev Saude Publica. 2015; 49.

6. Hoy D, Brooks P, Blyth F, Buchbinder R. The Epidemiology of low back pain. Best Pr Res Clin Rheumatol 2010; 24:769-781.

7. Manek NJ, MacGregor AJ. Epidemiology of back disorders: prevalence, risk factors, and prognosis. Curr Opin Intern Med 2005; 4(April):324-330.

8. Meziat Filho N, Silva GA. Invalidez por dor nas costas entre segurados da Previdência Social do Brasil. Rev Saude Publica 2011; 45(3):494-502.

9. Gouveia M, Augusto M. Custos indirectos da dor crónica em Portugal. Rev Port Saúde Pública. 2011; 29(2):100-107.

10. Deyo RA, Mirza SK, Turner JA, Martin BI. Overtreating Chronic Back Pain: Time to Back Off? Fam Med Heal CARE Syst . 2009; 22(1):62-68.

11. Krismer M, van Tulder M. Low back pain (non-specific). Best Pract Res Clin Rheumatol 2007; 21(1):77-91.

12. Carey TS, Freburger JK. Exercise and the prevention of low back pain: Ready for implementation. JAMA Intern Med 2016; 176(2):208-209.

13. Organização Pan-Americana de Saúde (OPAS). Prevenção de doenças crônicas, um investimento vital. Brasília: OPAS; 2005.

14. Scascighini L, Toma V, Dober-Spielmann S, Sprott H. Multidisciplinary treatment for chronic pain: a systematic review of interventions and outcomes. Rheumatology 2008; 47(5):670-678.

15. Oliveira IO, Pinto LLS, Oliveira MA, Cêra M. McKenzie method for low back pain. Rev Dor 2016; 17(4):303-306

16. Louw QA, Morris LD, Grimmer-Somers K. The Prevalence of low back pain in Africa: a systematic review. BMC Musculoskelet Disord 2007; 8:105.

17. Lim K-L, Jacobs P, Klarenbach S. A Population-Based Analysis of Healthcare Utilization of Persons With Back Disorders. Spine (Phila Pa 1976) 2006; 31(2):212-218. 
18. Ferreira GD, Silva MC, Rombaldi AJ, Wrege ED, Siqueira FV, Hallal PC. Prevalência de dor nas costas e fatores associados em adultos do sul do Brasil: estudo de base populacional. Brazilian J Phys Ther 2011; 15:31-36.

19. Amorim JSC, Silva SLA, Pereira LSM, Dias RC. Acesso aos serviços de fisioterapia e sua utilização por idosos com dor lombar. ConScientiae Saúde. 2014; 12(4):528-535.

20. Souza-Júnior PRB, Freitas MPS, Antonaci GDA, Szwarcwald CL. Desenho da amostra da Pesquisa Nacional de Saúde 2013. Epidemiol e Serviços Saúde 2015; 24(2):207-216.

21. Szwarcwald CL, Malta DC, Pereira CA, Vieira MLFP, Conde WL, Souza Júnior PRB, Damacena GN, Azevedo LO, Azevedo e Silva G, Theme Filha MM, Lopes CS, Romero DE, Almeida WS, Monteiro CA. Pesquisa Nacional de Saúde no Brasil: concepção e metodologia de aplicação. Cien Saude Colet 2014; 19(2):333342 .

22. Associação Brasileira de Empresas de Pesquisa (ABEP). Critério Brasil. São Paulo: ABEP; 2013.

23. Carey TS, Freburger JK, Holmes GM, Castel L, Darter J, Agans R, Kalsbeek W, Jackman A. A Long Way to Go. Spine (Phila Pa 1976) 2009; 34(7):718-724.

24. Mafi JN, McCarthy EP, Davis RB, Landon BE. Worsening trends in the management and treatment of back pain. JAMA Intern Med 2013; 173(17):1573-1581.

25. Balagué F, Mannion AF, Pellisé F, Cedraschi C. Non-specific low back pain. Lancet 2012; 379(9814):482-491.

26. Figueiredo VF, Pereira LSM, Ferreira PH, Pereira ADM, Amorim JSC. Incapacidade funcional, sintomas depressivos e dor lombar em idosos. Fisioter em Mov 2013; 26(Dl):549-557.

27. Wieser S, Horisberger B, Schmidhauser S, Eisenring C, Brügger U, Ruckstuhl A, Dietrich J, Mannion AF, Elfering A, Tamcan O, Müller U. Cost of low back pain in Switzerland in 2005. Eur J Heal Econ 2011; 12(5):455-467.

28. Martell BA, O'Connor PG, Kerns RD, Becker WC, Morales KH, Kosten TR, Fiellin DA. Systematic Review: Opioid Treatment for Chronic Back Pain: Prevalence, Efficacy, and Association with Addiction. Ann Intern Med 2007; 146(2):116.

29. Siqueira FV, Facchini LA, Hallal PC. Epidemiology of physiotherapy utilization among adults and elderly. Rev Saude Publica. 2005; 39(4):662-668.
30. Freburger JK, Carey TS, Holmes GM. Physical Therapy for Chronic Low Back Pain in North Carolina: Overuse, Underuse, or Misuse? Phys Ther 2011; 91(4):484-495.

31. Carey TS, Freburger JK, Holmes GM, Knauer S, Wallace A, Darter J. Race, care seeking and utilization for chronic back and neck pain: population perspectives. J Pain 2011; 11(4):343-350.

32. Viacava F, Bellido JG. Condições de saúde, acesso a serviços e fontes de pagamento, segundo inquéritos domiciliares. Cien Saude Colet 2016; 21(2):351-370.

33. Andrade MV, Noronha KVMS, Menezes RM, Souza MN, Reis CB, Martins DR, Gomes L. Desigualdade socioeconômica no acesso aos serviços de saúde no Brasil: um estudo comparativo entre as regiões brasileiras em 1998 e 2008. Econ Apl 2013; 17(4):623-645.

34. Jacobs P, Schopflocher D, Klarenbach S, Golmohammadi K, Ohinmaa A. A health production function for persons with back problems: results from the Canadian Community Health Survey of 2000. Spine (Phila Pa 1976) 2004; 29(20):2304-2308.

35. Côté P, Cassidy JD, Carroll L. The treatment of neck and low back pain: who seeks care? who goes where? Med Care 2001; 39(9):956-967.

36. Picavet HSJ, Struijs J, Westert G. Utilization of Health Resources due to Low Back Pain: Survey and Registered Data Compared. Spine (Phila Pa 1976) 2008; 33(4):436-444.

37. Kent PM, Keating JL. The epidemiology of low back pain in primary care. Chiropr Osteopat. 2005; 13:13.

Artigo apresentado em 07/11/2017

Aprovado em 13/04/2018

Versão final apresentada em 15/04/2018 